\title{
Some Results on Generalized Quasi-Einstein Manifolds
}

\author{
D. G. Prakasha ${ }^{1}$ and H. Venkatesha ${ }^{2}$ \\ ${ }^{1}$ Department of Mathematics, Karnatak University, Dharwad 580 003, India \\ ${ }^{2}$ Department of Mathematics, Kuvempu University, Shankaraghatta 577 451, India
}

Correspondence should be addressed to D. G. Prakasha; prakashadg@gmail.com

Received 19 September 2013; Accepted 31 October 2013; Published 21 January 2014

Academic Editors: Y. Fu, N. Tang, and H. Yan

Copyright (C) 2014 D. G. Prakasha and H. Venkatesha. This is an open access article distributed under the Creative Commons Attribution License, which permits unrestricted use, distribution, and reproduction in any medium, provided the original work is properly cited.

This paper deals with generalized quasi-Einstein manifold satisfying certain conditions on conharmonic curvature tensor. Here we study some geometric properties of generalized quasi-Einstein manifold and obtain results which reveal the nature of its associated 1 -forms.

\section{Introduction}

It is well known that a Riemannian or a semi-Riemannian manifold $\left(M^{n}, g\right)(n \geq 3)$ is an Einstein manifold if its Ricci tensor $S$ of type $(0,2)$ is of the form $S=(r / n) g, r$ being the (constant) scalar curvature of the manifold. Let $U_{S_{1}}=\{x \in$ $M: S \neq(r / n) g$ at $x\}$. Then the manifold $\left(M^{n}, g\right)$ is said to be a quasi-Einstein manifold [1-7] if, on $U_{S_{1}} \subset M$, we have

$$
S=\alpha g+\beta A \otimes A,
$$

where $A$ is 1 -form on $U_{S_{1}}$ and $\alpha, \beta$ are some smooth functions on $U_{S_{1}}$. It is clear that the function $\beta$ and the 1 -form $A$ are nonzero at every point on $U_{S_{1}}$. The scalars $\alpha, \beta$ are the associated scalars of the manifold. Also the 1-form $A$ is called associated 1-form of the manifold defined by $g(X, \rho)=A(X)$ for any vector field $X, \rho$ being a unit vector field called generator of the manifold. Such an $n$-dimensional quasiEinstein manifold is denoted by $(Q E)_{n}$. The quasi-Einstein manifolds have also been studied in [8-11].

Generalizing the notion of quasi-Einstein manifold, in [12], De and Ghosh introduced the notion of generalized quasi-Einstein manifolds and studied its geometrical properties with the existence of such notion by several nontrivial examples. A Riemannian manifold $\left(M^{n}, g\right)(n>3)$ is said to be generalized quasi-Einstein manifold if its Ricci tensor $S$ of type $(0,2)$ is not identically zero and satisfies the condition:

$$
S(X, Y)=\alpha g(X, Y)+\beta A(X) A(Y)+\gamma B(X) B(Y),
$$

where $\alpha, \beta$, and $\gamma$ are scalars of which $\beta \neq 0, \gamma \neq 0$ and $A, B$ are nowhere vanishing 1-forms such that $g(X, \rho)=A(X)$, $g(X, \mu)=B(X)$ for any vector field $X$. The unit vectors $\rho$ and $\mu$ corresponding to 1 -forms $A$ and $B$ are orthogonal to each other. Also $\rho$ and $\mu$ are the generators of the manifold. Such an $n$-dimensional manifold is denoted by $G(Q E)_{n}$. The generalized quasi-Einstein manifolds have also been studied in $[13-16]$.

In 2008, De and Gazi [17] introduced the notion of nearly quasi-Einstein manifolds. A nonflat Riemannian manifold $\left(M^{n}, g\right)(n>2)$ is called a nearly quasi-Einstein manifold if its Ricci tensor of type $(0,2)$ is not identically zero and satisfies the condition:

$$
S(X, Y)=\alpha g(X, Y)+\beta E(X, Y),
$$

where $\alpha$ and $\beta$ are nonzero scalars and $E$ is a nonzero symmetric tensor of type $(0,2)$. An $n$-dimensional nearly quasi-Einstein manifold was denoted by $N(Q E)_{n}$. The nearly quasi-Einstein manifolds have also been studied by Prakasha and Bagewadi [18].

The present paper is organized as follows. Section 2 deals with the preliminaries. Section 3 is concerned with conharmonic curvature tensor on $G(Q E)_{n}$. In this section, it is proved that a conharmonically flat $G(Q E)_{n}$ is one of the manifold of generalized quasiconstant curvature. Also, it is proved that if in a $G(Q E)_{n}(n \geq 3)$ the associated scalars are constants and the generators $\rho$ and $\mu$ are vector 
fields with the associated 1-forms $A$ and $B$ not being the 1forms of recurrences, then the manifold is conharmonically conservative. In Section 4, we consider $G(Q E)_{n}(n \geq 3)$ satisfying the condition $\mathscr{L} \cdot S=0$. In the last section we study some geometrical properties of a $G(Q E)_{n}$.

\section{Preliminaries}

Consider a $G(Q E)_{n}$ with associated scalars $\alpha, \beta$, and $\gamma$ and associated 1 -forms $A, B$. Then from (2) we get

$$
r=n \alpha+\beta+\gamma
$$

where $r$ is the scalar curvature of the manifold. Since $\rho$ and $\mu$ are orthogonal unit vector fields, $g(\rho, \rho)=1, g(\mu, \mu)=1$, and $g(\rho, \mu)=0$. Again from (2), we have

$$
\begin{array}{ll}
S(X, \rho)=(\alpha+\beta) A(X), & S(\rho, \rho)=\alpha+\beta, \\
S(X, \mu)=(\alpha+\gamma) B(X), & S(\mu, \mu)=\alpha+\gamma .
\end{array}
$$

Let $Q$ be the symmetric endomorphism of the tangent space at each point of the manifold corresponding to the Ricci tensor $S$. Then $g(Q X, Y)=S(X, Y)$ for all $X, Y$.

The rank-four tensor $\mathscr{L}^{\prime}$ that remains invariant under conharmonic transformation for an $n$-dimensional Riemannian manifold is given by [19]

$$
\begin{aligned}
\mathscr{L}^{\prime}(X, Y, Z, U) \\
=R^{\prime}(X, Y, Z, U)-\frac{1}{n-2} \\
\quad \times[S(Y, Z) g(X, U)-S(X, Z) g(Y, U) \\
\quad+g(Y, Z) S(X, U)-g(X, Z) S(Y, U)],
\end{aligned}
$$

where $\mathscr{L}^{\prime}(X, Y, Z, U)=g(\mathscr{L}(X, Y) Z, U)$ and $R^{\prime}$ denotes the Riemannian curvature tensor of type $(0,4)$ defined by $R^{\prime}(X, Y, Z, U)=g(R(X, Y) Z, U)$, where $R$ is the Riemannian curvature tensor of type $(1,3)$.

\section{Conharmonic Curvature Tensor on $G(Q E)_{n}$}

A manifold of generalized quasiconstant curvature tensor is $G(Q E)_{n}$. But the converse is not true, in general. In this section, we enquire under what conditions the converse will be true.

A manifold whose conharmonic curvature tensor vanishes at every point of the manifold is called conharmonically flat manifold. Thus this tensor represents the deviation of the manifold from conharmonic flatness. It satisfies all the symmetric properties of the Riemannian curvature tensor $R^{\prime}$. There are many physical applications of the tensor $\mathscr{L}$. For example, in [20], Abdussattar showed that sufficient condition for a space-time to be conharmonic to a flat is either empty in which case it is flat or filled with a distribution represented by energy momentum tensor $T$ possessing the algebraic structure of an electromagnetic field and conformal to a flat space-time [20].
Let us consider that the manifold under consideration is conharmonically flat. Then from (6) we have

$$
\begin{aligned}
R^{\prime}(X, Y, Z, U) & \\
=\frac{1}{n-2}[ & S(Y, Z) g(X, U)-S(X, Z) g(Y, U) \\
& \quad+g(Y, Z) S(X, U)-g(X, Z) S(Y, U)] .
\end{aligned}
$$

Using (2) in (7), we obtain

$$
\begin{aligned}
& R^{\prime}(X, Y, Z, U) \\
& =\frac{2 \alpha}{n-2}[g(Y, Z) g(X, U)-g(X, Z) g(Y, U)] \\
& +\frac{\beta}{n-2}[g(X, U) A(Y) A(Z)-g(Y, U) A(X) A(Z) \\
& \quad+g(Y, Z) A(X) A(U)-g(X, Z) A(Y) A(U)] \\
& +\frac{\gamma}{n-2}[g(X, U) B(Y) B(Z)-g(Y, U) B(X) B(Z) \\
& \quad+g(Y, Z) B(X) B(U)-g(X, Z) B(Y) B(U)] .
\end{aligned}
$$

According to Chen and Yano [21], a Riemannian manifold $\left(M^{n}, g\right)(n>3)$ is said to be of quasiconstant curvature if it is conformally flat and its curvature tensor $R^{\prime}$ of type $(0,4)$ has the form:

$$
\begin{aligned}
R^{\prime}(X, Y, Z, U) & \\
= & p[g(Y, Z) g(X, U)-g(X, Z) g(Y, U)] \\
+ & q[g(X, U) T(Y) T(Z)-g(Y, U) T(X) T(Z) \\
& \quad+g(Y, Z) T(X) T(U)-g(X, Z) T(Y) T(U)],
\end{aligned}
$$

where $T$ is a form defined by $g(X, \rho)=A(X)$ with $\rho$ as a unit vector field and $p, q$ are scalars of which $q \neq 0$.

In [12], De and Ghosh generalize the notion of quasiconstant curvature and prove the existence of such a manifold. A Riemannian manifold is said to be a manifold of generalized quasiconstant curvature, if the curvature tensor $R^{\prime}$ of type $(0,4)$ satisfies the condition:

$$
\begin{aligned}
R^{\prime}(X, Y, Z, U) & \\
= & p[g(Y, Z) g(X, U)-g(X, Z) g(Y, U)] \\
+ & q[g(X, U) T(Y) T(Z)-g(Y, U) T(X) T(Z) \\
& +g(Y, Z) T(X) T(U)-g(X, Z) T(Y) T(U)] \\
+ & s[g(X, U) D(Y) D(Z)-g(Y, U) D(X) D(Z) \\
& +g(Y, Z) D(X) D(U)-g(X, Z) D(Y) D(U)],
\end{aligned}
$$

where $p, q$, and $s$ are scalars and $T$ and $D$ are nonzero 1 -forms. 
We assume that the unit vector fields $\rho$ and $\rho_{1}$ defined by $g(X, \rho)=T(X)$ and $g\left(X, \rho_{1}\right)=D(X)$ are orthogonal; that is, $g\left(\rho, \rho_{1}\right)=0$. Now the relation (8) can be written as

$$
\begin{aligned}
R^{\prime}(X, Y, Z, U) \\
=p_{1}[g(Y, Z) g(X, U)-g(X, Z) g(Y, U)] \\
+q_{1}[g(X, U) T(Y) T(Z)-g(Y, U) T(X) T(Z) \\
+g(Y, Z) T(X) T(U)-g(X, Z) T(Y) T(U)] \\
+s_{1}[g(X, U) D(Y) D(Z)-g(Y, U) D(X) D(Z) \\
+g(Y, Z) D(X) D(U)-g(X, Z) D(Y) D(U)],
\end{aligned}
$$

where $p_{1}=(2 \alpha /(n-2)), q_{1}=(\beta /(n-2))$, and $s_{1}=(\gamma /(n-$ $2))$. Comparing (10) and (11), it follows that the manifold is of generalized quasiconstant curvature. Thus we have the following theorem.

Theorem 1. A conharmonically flat $G(Q E)_{n}$ is one of generalized quasiconstant curvature.

Next, differentiating (6) covariantly and then contracting we obtain

$$
\begin{aligned}
(\operatorname{div} \cdot \mathscr{L})(X, Y) Z \\
=(\operatorname{div} \cdot R)(X, Y) Z-\frac{1}{n-2}\left[\left(\nabla_{X} S\right)(Y, Z)-\left(\nabla_{Y} S\right)(X, Z)\right] \\
\quad-\frac{1}{2(n-2)}\left[\left(\nabla_{X} r\right) g(Y, Z)-\left(\nabla_{Y} r\right) g(X, Z)\right],
\end{aligned}
$$

where div denotes the divergence.

Again, it is known that in a Riemannian manifold we have

$$
(\operatorname{div} \cdot R)(X, Y) Z=\left(\nabla_{X} S\right)(Y, Z)-\left(\nabla_{Y} S\right)(X, Z) \text {. }
$$

Consequently by virtue of (13), the relation (12) takes the form:

$$
\begin{aligned}
(\operatorname{div} \cdot \mathscr{L})(X, Y) Z \\
=\frac{n-3}{n-2}\left[\left(\nabla_{X} S\right)(Y, Z)-\left(\nabla_{Y} S\right)(X, Z)\right] \\
\quad-\frac{1}{2(n-2)}\left[\left(\nabla_{X} r\right) g(Y, Z)-\left(\nabla_{Y} r\right) g(X, Z)\right] .
\end{aligned}
$$

Now consider the associated scalars $\alpha, \beta$, and $\gamma$ as constants; then (4) yields that the scalar curvature $r$ is constant, and hence $d r(X)=0$ for all $X$. Consequently, (14) reduces to

$$
(\operatorname{div} \cdot \mathscr{L})(X, Y) Z=\frac{n-3}{n-2}\left[\left(\nabla_{X} S\right)(Y, Z)-\left(\nabla_{Y} S\right)(X, Z)\right] .
$$

Since $\alpha, \beta$, and $\gamma$ are constants, we have from (2) that

$$
\begin{aligned}
\left(\nabla_{X} S\right)(Y, Z)= & \beta\left[\left(\nabla_{X} A\right)(Y) A(Z)+A(Y)\left(\nabla_{X} A\right)(Z)\right] \\
& +\gamma\left[\left(\nabla_{X} A\right)(Y) A(Z)+A(Y)\left(\nabla_{X} A\right)(Z)\right] .
\end{aligned}
$$

By virtue of (16), we get from (15) that

$$
\begin{aligned}
& (\operatorname{div} \cdot \mathscr{L})(X, Y) Z \\
& =\frac{n-3}{n-2}\left[\beta \left\{\left(\nabla_{X} A\right)(Y) A(Z)+A(Y)\left(\nabla_{X} A\right)(Z)\right.\right. \\
& \left.\left.\quad-\left(\nabla_{Y} A\right)(X) A(Z)-A(X)\left(\nabla_{Y} A\right)(Z)\right\}\right] \\
& +\gamma\left\{\left(\nabla_{X} B\right)(Y) B(Z)+B(Y)\left(\nabla_{X} B\right)(Z)\right. \\
& \left.\quad-\left(\nabla_{Y} B\right)(X) B(Z)-B(X)\left(\nabla_{Y} B\right)(Z)\right\} .
\end{aligned}
$$

Next, if the generators $\rho$ and $\mu$ of the manifold under consideration are recurrent vector fields [22], then we have $\nabla_{X} \rho=\pi_{1}(X) \rho$ and $\nabla_{X} \mu=\pi_{2}(X) \mu$, where $\pi_{1}$ and $\pi_{2}$ are the $1-$ forms of the recurrence such that $\pi_{1}$ and $\pi_{2}$ are different from $A$ and $B$. Consequently, we get

$$
\begin{aligned}
& \left(\nabla_{X} A\right)(Y)=g\left(\nabla_{X} \rho, Y\right)=g\left(\pi_{1}(X) \rho, Y\right)=\pi_{1}(Y) A(Z), \\
& \left(\nabla_{X} B\right)(Y)=g\left(\nabla_{X} \mu, Y\right)=g\left(\pi_{2}(X) \mu, Y\right)=\pi_{2}(Y) B(Z) .
\end{aligned}
$$

In view of (18), relation (17) reduces to

$(\operatorname{div} \cdot \mathscr{L})(X, Y) Z$

$$
\begin{aligned}
=\frac{2(n-3)}{(n-2)}[ & \beta\left\{\pi_{1}(X) A(Y)-\pi_{1}(Y) A(X)\right\} A(Z) \\
& \left.\times \gamma\left\{\pi_{2}(X) B(Y)-\pi_{2}(Y) B(X)\right\} B(Z)\right] .
\end{aligned}
$$

Also, since $g(\rho, \rho)=g(\mu, \mu)=1$, it follows that $\left(\nabla_{X} A\right)(\rho)=$ $g\left(\nabla_{X} \rho, \rho\right)=0$, and hence $(18)$ reduces to $\pi_{1}(X)=0$ for all $X$. Similarly, we have $\pi_{2}(X)=0$. Hence, from (19), we have $(\operatorname{div} \cdot \mathscr{L})(X, Y) Z=0$; that is, the manifold under consideration is conharmonically conservative. Hence, we can state the following theorem.

Theorem 2. If, in a $G(Q E)_{n}(n>3)$, the associated scalars are constants and the generators $\rho$ and $\mu$ corresponding to the associated 1-forms $A$ and $B$ are not being the recurrence 1forms, then the manifold is conharmonically conservative.

\section{4. $G(Q E)_{n}(n>3)$ Satisfying}

\section{the Condition $\mathscr{L} \cdot S=0$}

Let us consider a $G(Q E)_{n}(n>3)$, satisfying the condition $\mathscr{L} \cdot S=0$. Then we have

$$
S(\mathscr{L}(X, Y) Z, U)+S(Z, \mathscr{L}(X, Y) U)=0 .
$$

Setting $Z=\rho$ and $U=\mu$ in (20), we have

$$
S(\mathscr{L}(X, Y) \rho, \mu)+S(\rho, \mathscr{L}(X, Y) \mu)=0 .
$$

By using (5) in (21), we obtain

$$
(\alpha+\gamma) B(\mathscr{L}(X, Y) \rho)+(\alpha+\beta) A(\mathscr{L}(X, Y) \mu)=0 .
$$


Next, putting $Z=\rho$ in (6) and then taking inner product with $\mu$, we get

$$
\begin{aligned}
\mathscr{L}^{\prime}(X, Y, \rho, \mu) \\
=R^{\prime}(X, Y, \rho, \mu)-\frac{1}{n-2} \\
\times[S(Y, \mu) g(X, \rho)-S(X, \mu) g(Y, \rho) \\
\quad+g(Y, \mu) S(X, \rho)-g(X, \mu) S(Y, \rho)],
\end{aligned}
$$

where $g(\mathscr{L}(X, Y) \rho, \mu)=\mathscr{L}^{\prime}(X, Y, \rho, \mu)=B(\mathscr{L}(X, Y) \rho)$. Again from (5) we obtain from (23)

$$
\begin{aligned}
\mathscr{L}^{\prime}(X, Y, \rho, \mu)= & R^{\prime}(X, Y, \rho, \mu) \\
& -\frac{2 \alpha+\beta+\gamma}{(n-2)}[A(Y) B(X)-A(X) B(Y)] .
\end{aligned}
$$

Again, plugging $Z=\mu$ in (6) and then taking inner product with $\rho$, we have

$$
\begin{aligned}
\mathscr{L}^{\prime}(X, Y, \mu, \rho)= & R^{\prime}(X, Y, \mu, \rho) \\
& -\frac{2 \alpha+\beta+\gamma}{(n-2)}[A(X) B(Y)-A(Y) B(X)],
\end{aligned}
$$

where $g(\mathscr{L}(X, Y) \mu, \rho)=\mathscr{L}^{\prime}(X, Y, \mu, \rho)=A(\mathscr{L}(X, Y) \mu)$. From (24) and (25), one can get

$$
A(\mathscr{L}(X, Y) \mu)=-B(\mathscr{L}(X, Y) \rho) .
$$

By taking account of (26) in (22), we obtain

$$
(\gamma-\beta) B(\mathscr{L}(X, Y) \mu)=0 .
$$

This implies either $\gamma=\beta$ or $B(\mathscr{L}(X, Y) \mu)=0$.

Now if $\gamma=\beta$, then, from (2), we have

$$
\begin{aligned}
S(X, Y) & =\alpha g(X, Y)+\beta[A(X) A(Y)+B(X) B(Y)] \\
& =\alpha g(X, Y)+\beta E(X, Y),
\end{aligned}
$$

where $E(X, Y)=A(X) A(Y)+B(X) B(Y)$. That is, the manifold is a $N(Q E)_{n}$.

On the other hand, if $B(L(X, Y) \rho)=0$, then (24) gives

$$
R^{\prime}(X, Y, \rho, \mu)=\frac{(2 \alpha+\beta+\gamma)}{(n-2)}[A(Y) B(X)-A(X) B(Y)]
$$

Thus we can state the following theorem.

Theorem 3. If $M$ is a $G(Q E)_{n}(n>3)$ satisfying the condition $\mathscr{L} \cdot S=0$, then either $M$ is $N(Q E)_{n}$ or the curvature tensor $R$ of the manifold satisfies the property (29).

\section{Some Geometric Properties of $G(Q E)_{n}$}

In [23], Gray introduced two classes of Riemannian manifolds determined by the covariant differentiation of Ricci tensor. Class A consists of all Riemannian manifolds whose Ricci tensor $S$ satisfies the condition:

$$
\left(\nabla_{X} S\right)(Y, Z)=\left(\nabla_{Y} S\right)(X, Z)
$$

that is, Ricci tensor $S$ is a Codazzi type tensor.

Class B consists of all Riemannian manifolds whose Ricci tensor $S$ satisfies the condition:

$$
\left(\nabla_{X} S\right)(Y, Z)+\left(\nabla_{Y} S\right)(Z, X)+\left(\nabla_{Z} S\right)(X, Y)=0
$$

that is, Ricci tensor $S$ is cyclic parallel.

First suppose that the associated scalars are constants and the Ricci tensor $S$ is of Codazzi type. Then from (2) we obtain

$$
\begin{aligned}
\left(\nabla_{X} S\right)(Y, Z)= & \beta\left[\left(\nabla_{X} A\right)(Y) A(Z)+\left(\nabla_{X} A\right)(Z) A(Y)\right] \\
& +\gamma\left[\left(\nabla_{X} B\right)(Y) B(Z)+\left(\nabla_{X} B\right)(Z) B(Y)\right] .
\end{aligned}
$$

Interchanging $X$ and $Y$ in (32), we have

$$
\begin{aligned}
\left(\nabla_{Y} S\right)(X, Z)= & \beta\left[\left(\nabla_{Y} A\right)(X) A(Z)+\left(\nabla_{Y} A\right)(Z) A(X)\right] \\
& +\gamma\left[\left(\nabla_{Y} B\right)(X) B(Z)+\left(\nabla_{Y} B\right)(Z) B(X)\right] .
\end{aligned}
$$

Since $S$ is of Codazzi type, we have from (32) and (33) that

$$
\begin{aligned}
\beta\left[\left(\nabla_{X} A\right)(Y) A(Z)+\left(\nabla_{X} A\right)(Z) A(Y)\right] \\
+\gamma\left[\left(\nabla_{X} B\right)(Y) B(Z)+\left(\nabla_{X} B\right)(Z) B(Y)\right] \\
=\beta\left[\left(\nabla_{Y} A\right)(X) A(Z)+\left(\nabla_{Y} A\right)(Z) A(X)\right] \\
+\gamma\left[\left(\nabla_{Y} B\right)(X) B(Z)+\left(\nabla_{Y} B\right)(Z) B(X)\right] .
\end{aligned}
$$

Putting $Z=\rho$ in (34) and using $\left(\nabla_{X} A\right)(\rho)=0$ and $\left(\nabla_{X} B\right)(\rho)=0$, since $\rho$ is a unit vector, we get

$$
\left(\nabla_{X} B\right)(Y)-\left(\nabla_{Y} B\right)(X)=0 .
$$

This shows that $d B(X, Y)=0$.

Again, putting $Z=\mu$ in (34) and using $\left(\nabla_{X} A\right)(\mu)=0$ and $\left(\nabla_{X} B\right)(\mu)=0$, since $\mu$ is a unit vector, we get

$$
\left(\nabla_{X} A\right)(Y)-\left(\nabla_{Y} A\right)(X)=0 .
$$

This shows that $d A(X, Y)=0$.

Thus we can state the following theorem.

Theorem 4. In a $G(Q E)_{n}$, if the associated scalars are constants and the Ricci tensor is of Codazzi type, then the associated 1-forms $A$ and $B$ are closed.

Next, suppose that the generators $\rho$ and $\mu$ are Killing vector fields in a $G(Q E)_{n}$ and the associated scalars are constants. Then

$$
\left(\mathbb{L}_{U} g\right)(X, Y)=0, \quad\left(\mathbb{L}_{V} g\right)(X, Y)=0,
$$


where $\mathbb{L}$ denotes the Lie derivative, which implies that

$$
\begin{aligned}
& g\left(\nabla_{X} U, Y\right)+g\left(X, \nabla_{Y} U\right)=0, \\
& g\left(\nabla_{X} V, Y\right)+g\left(X, \nabla_{Y} V\right)=0 .
\end{aligned}
$$

From (38) it follows that

$$
\begin{aligned}
& \left(\nabla_{X} A\right)(Y)+\left(\nabla_{Y} A\right)(X)=0 \\
& \left(\nabla_{X} B\right)(Y)+\left(\nabla_{Y} B\right)(X)=0
\end{aligned}
$$

since $g\left(\nabla_{X} U, Y\right)=\left(\nabla_{X} A\right)(Y)$ and $g\left(\nabla_{X} V, Y\right)=\left(\nabla_{X} B\right)(Y)$.

Similarly, we have

$$
\begin{aligned}
& \left(\nabla_{X} A\right)(Z)+\left(\nabla_{Z} A\right)(X)=0, \\
& \left(\nabla_{X} B\right)(Z)+\left(\nabla_{Z} B\right)(X)=0, \\
& \left(\nabla_{Y} A\right)(Z)+\left(\nabla_{Z} A\right)(Y)=0, \\
& \left(\nabla_{Y} B\right)(Z)+\left(\nabla_{Z} B\right)(Y)=0
\end{aligned}
$$

for all $X, Y$, and $Z$. Now from (2) and using the relations (39)(40) we have

$$
\left(\nabla_{X} S\right)(Y, Z)+\left(\nabla_{Y} S\right)(Z, X)+\left(\nabla_{Z} S\right)(X, Y)=0 .
$$

Therefore we can state the following theorem.

Theorem 5. In a $G(Q E)_{n}$, if the generators are Killing vector fields and the associated scalars are constants, then the Ricci tensor of the manifold is cyclic parallel.

\section{Conflict of Interests}

The authors declare that there is no conflict of interests regarding the publication of this paper.

\section{Acknowledgment}

D. G. Prakasha is thankful to University Grants Commission, New Delhi, India, for financial support in the form of Major Research Project (no. 39-30/2010 (SR), dated December 23, 2012).

\section{References}

[1] M. C. Chaki and R. K. Maity, "On quasi Einstein manifolds," Publicationes Mathematicae, vol. 57, no. 3-4, pp. 297-306, 2000.

[2] F. Defever, R. Deszcz, M. Hotlos, M. Kucharski, and Z. Senturk, "Generalisations of Robertson walker space, Annales Universitatis Scientiarum Budapestinensis de Rolando Eotvos Nominatae," Sectio Mathematica, vol. 43, pp. 13-24, 2000.

[3] R. Deszcz, F. Dillen, L. Verstraelen, and L. Vrancken, "QuasiEinstein totally real submanifolds of the nearly Kähler 6sphere," Tohoku Mathematical Journal, vol. 51, no. 4, pp. 461478, 1999.

[4] R. Deszcz, M. Glogowska, M. Hotlo's, and Z. Senturk, "On certain quasi-Einstein semi-symmetric hypersurfaces," Annales Universitatis Scientarium Budapestinensis, vol. 41, pp. 153-166, 1998.
[5] R. Deszcz, M. Hotlo's, and Z. Senturk, "Quasi-Einstein hypersurfaces in semi-Riemannian space forms," Colloquium Mathematicum, vol. 81, pp. 81-97, 2001.

[6] R. Deszcz, M. Hotlo's, and Z. Senturk, "On curvature properties of quasi-Einatein hypersurfaces in semi-Euclidean spaces," Soochow Journal of Mathematics, vol. 27, no. 4, pp. 375-389, 2001.

[7] M. Głogowska, "On quasi-Einstein Cartan type hypersurfaces," Journal of Geometry and Physics, vol. 58, no. 5, pp. 599-614, 2008.

[8] M. C. Chaki and P. K. Ghoshal, "Some global properties of quasi Einstein manifolds," Publicationes Mathematicae, vol. 63, no. 4, pp. 635-641, 2003.

[9] U. C. De and G. C. Ghosh, "On quasi Einstein manifolds," Periodica Mathematica Hungarica, vol. 48, no. 1-2, pp. 223-231, 2004.

[10] A. A. Shaikh and A. Patra, "On quasi-conformally at quasiEinstein spaces," Differential Geometry-Dynamical Systems, vol. 12, pp. 201-212, 2010.

[11] A. A. Shaikh, D. W. Yoon, and S. K. Hui, "On quasi-Einstein spacetimes," Tsukuba Journal of Mathematics, vol. 33, no. 2, pp. 305-326, 2009.

[12] U. C. De and G. C. Ghosh, "On generalized quasi-Einstein manifolds," Kyungpook Mathematical Journal, vol. 44, pp. 607615, 2004.

[13] M. C. Chaki, “On generalized quasi Einstein manifolds," Publicationes Mathematicae, vol. 58, no. 4, pp. 683-691, 2001.

[14] U. C. De and S. Mallick, "On the existence of generalized quasiEinstein manifolds, Archivum Mathematicum (Brno)," Tomus, vol. 47, pp. 279-291, 2011.

[15] S. K. Hui and R. S. Lemence, "On generalized quasi-Einstein manifold admitting W2- curvature tensor," International Journal of Mathematical Analysis, vol. 6, no. 23, pp. 1115-1121, 2012.

[16] A. A. Shaikh and S. K. Hui, "On some classes of generalized quasi-Einstein manifolds," Communications of the Korean Mathematical Society, vol. 24, no. 3, pp. 415-424, 2009.

[17] U. C. De and A. K. Gazi, "On nearly quasi-Einstein manifolds," Novi Sad Journal of Mathematics, vol. 38, no. 2, pp. 115-121, 2008.

[18] D. G. Prakasha and C. S. Bagewadi, "On nearly quasi-Einstein manifolds," Mathematica Pannonica, vol. 21, no. 2, pp. 265-273, 2010.

[19] Y. Ishii, “On conharmonic transformations," Tensor. N.S., vol. 7, pp. 73-80, 1957.

[20] D. B. Abdussattar, "On conharmonic transformations in general relativity," Bulletin of Calcutta Mathematical Society, vol. 41, pp. 409-416, 1966.

[21] B. Y. Chen and K. Yano, "Hypersurfaces of conformally at spaces," Tensor. N. S., vol. 26, pp. 318-322, 1972.

[22] S. Tanno, "Ricci curvatures of Riemannian manifolds," Tsukuba Journal of Mathematics, vol. 40, pp. 441-448, 1988.

[23] A. Gray, "Einstein-like manifolds which are not Einstein," Geometriae Dedicata, vol. 7, no. 3, pp. 259-280, 1978. 


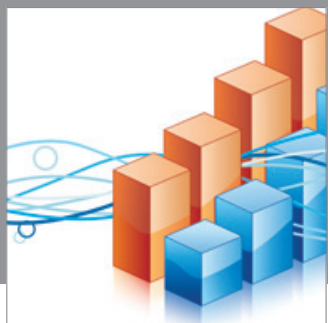

Advances in

Operations Research

mansans

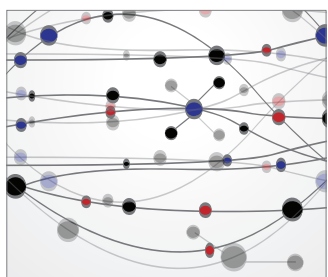

The Scientific World Journal
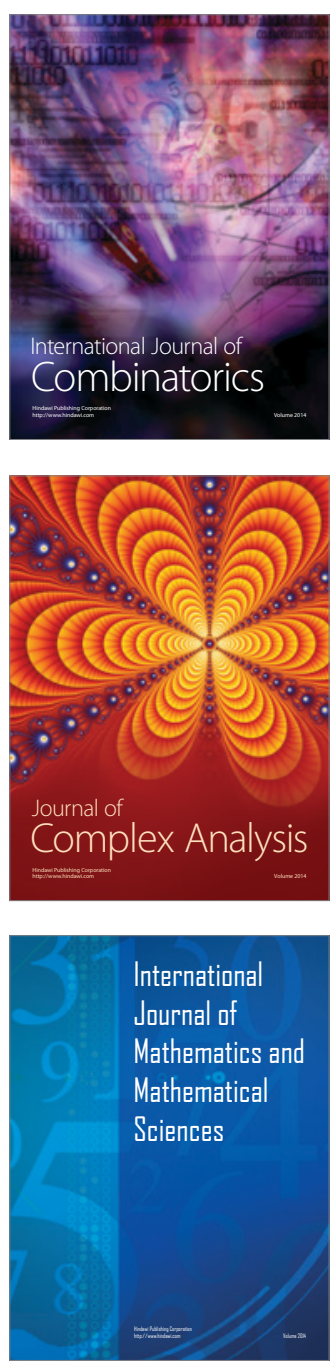
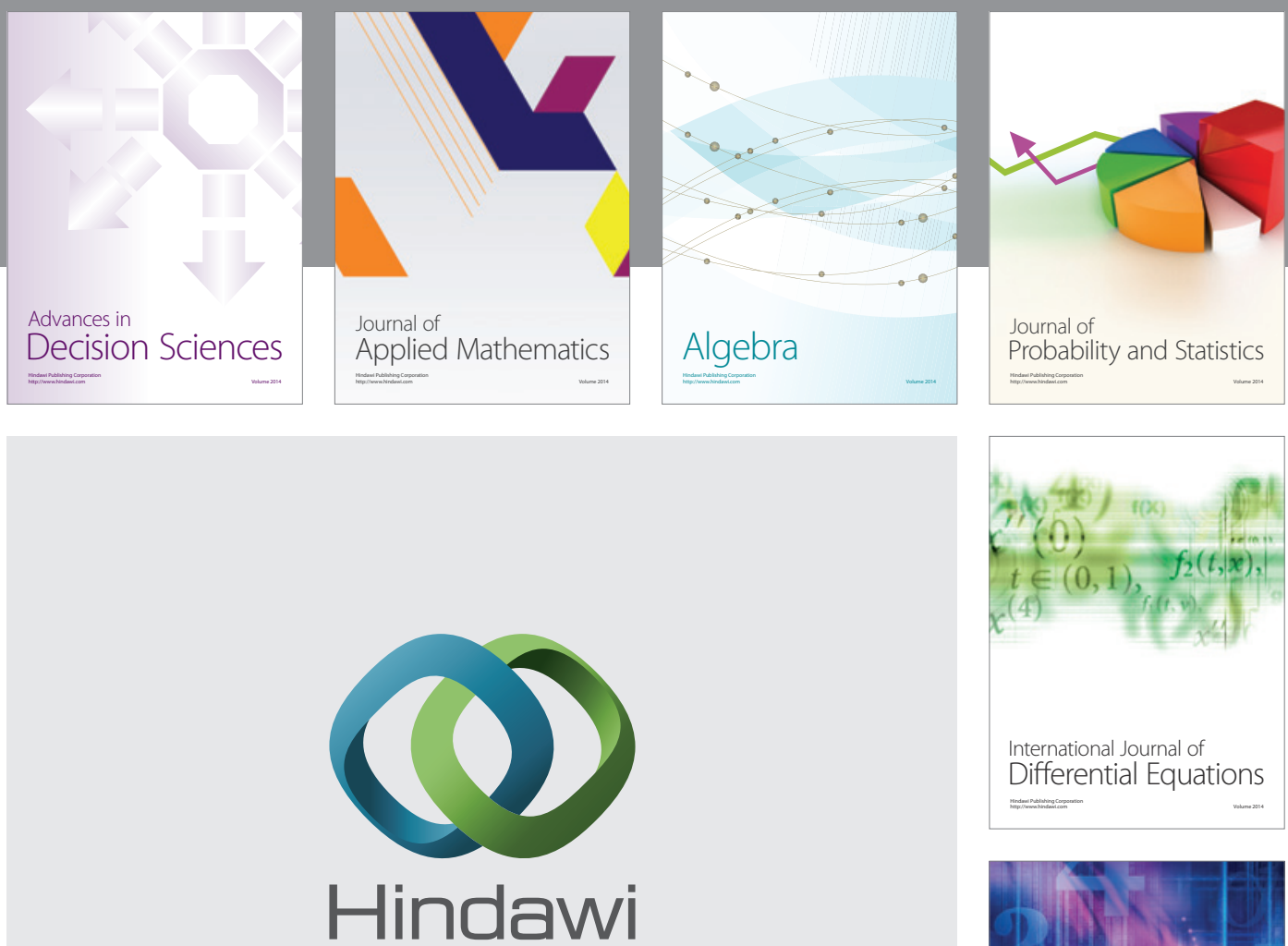

Submit your manuscripts at http://www.hindawi.com
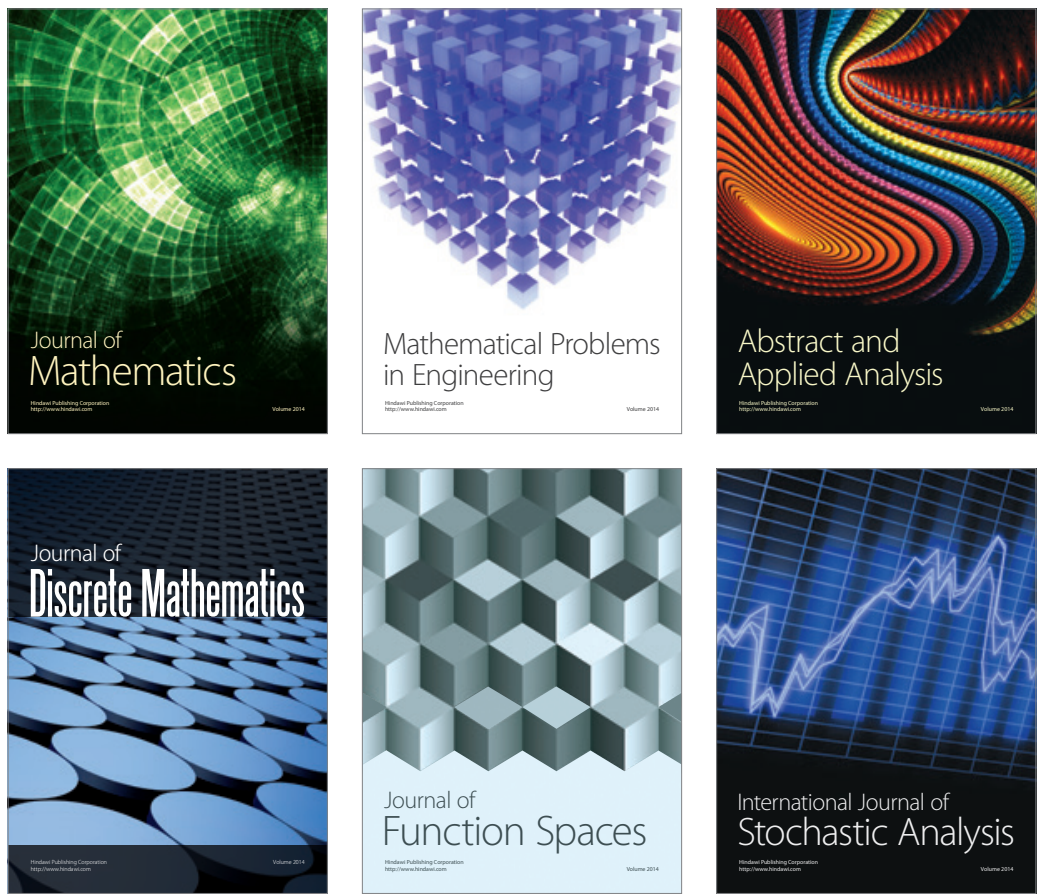

Journal of

Function Spaces

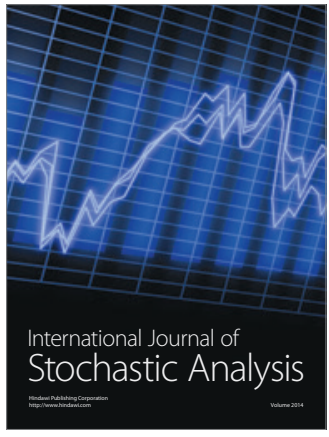

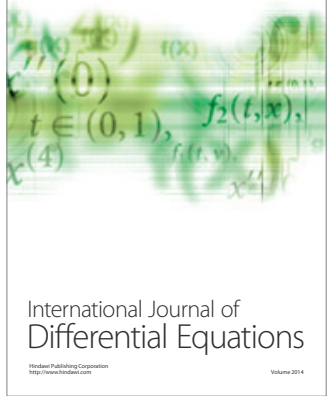
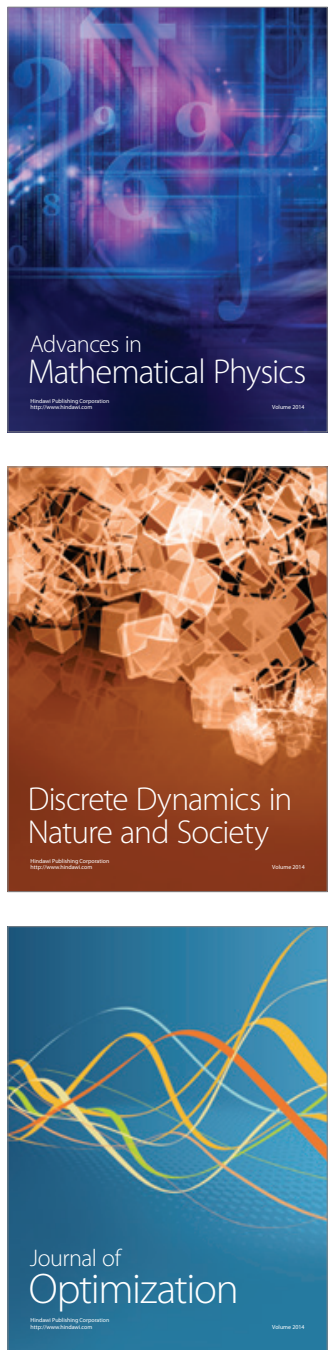